\title{
ARTICLE \\ Podoplanin mediates the renoprotective effect of berberine on diabetic kidney disease in mice
}

\author{
Jing $\mathrm{Yu}^{1}$, Guan-nan Zong ${ }^{2}$, Hao $\mathrm{Wu}^{3}$ and Ke-qin Zhang ${ }^{2}$
}

\begin{abstract}
Hyperglycemia-caused podocyte injury plays a crucial role in the progress of diabetic kidney disease. Podoplanin, one of the podocyte-associated molecules, is closely related to the integrity of the glomerular filtration barrier. A number of studies demonstrate that berberine could ameliorate renal dysfunction in diabetic mice with nephropathy, but the molecular mechanisms have not been fully elucidated. In this study, we explored the relationship between the renoprotective effect of berberine and podoplanin expression in streptozotocin (STZ)-induced diabetic mice as well as mouse podocytes (MPC5 cells) cultured in high glucose $(\mathrm{HG}, 30 \mathrm{mM})$ medium. We found that the expression levels of podoplanin were significantly decreased both in the renal glomerulus of STZ-induced diabetic mice and HG-cultured MPC5 cells. We also demonstrated that NF-KB signaling pathway was activated in MPC5 cells under HG condition, which downregulated the expression level of podoplanin, thus leading to increased podocyte apoptosis. Administration of berberine (100, $200 \mathrm{mg} / \mathrm{kg}$ every day, ig, for 8 weeks) significantly improved hyperglycemia and the renal function of STZ-induced diabetic mice and restored the expression level of podoplanin in renal glomerulus. In high glucose-cultured MPC5 cells, treatment with berberine (30-120 $\mu \mathrm{M})$ dose-dependently decreased the apoptosis rate, increased the expression of podoplanin, and inhibited the activation of NF-KB signaling pathway. When podoplanin expression was silenced with shRNA, berberine treatment still inhibited the NF-KB signaling pathway, but its antiapoptotic effect on podocytes almost disappeared. Our results suggest that berberine inhibits the activation of NF-KB signaling pathway, thus increasing the podoplanin expression to exert renoprotective effects.
\end{abstract}

Keywords: podoplanin; berberine; diabetic kidney disease; renoprotective effects; podocytes; apoptosis

Acta Pharmacologica Sinica (2019) 40:1544-1554; https://doi.org/10.1038/s41401-019-0263-3

\section{INTRODUCTION}

Diabetic kidney disease (DKD), one of the most serious microvascular complications of diabetes mellitus, is the leading cause of end-stage renal disease (ESRD) [1]. Clinically, DKD is characterized by persistent abnormal urine albumin excretion and progressive decline of the glomerular filtration rate (GFR) [2]. At present, the specific pathogenesis of DKD has not been fully elucidated. Growing evidence has demonstrated that glomerular podocyte injury may play an important role in the pathogenesis of DKD [3-5]. Glomerular visceral epithelial cells, called podocytes, are highly specialized cells in the kidney that constitute the outermost layer of the glomerular filtration barrier. Further study of podocytes has shown that podocyte-associated molecules, such as nephrin [6, 7], podocin [8], and podocalyxin [9], are closely related to the development of DKD. For podoplanin, another podocyte-associated molecule, its relationship with DKD remains unknown.

Podoplanin (PDPN), a membrane protein on podocytes, was first reported by Breiteneder-Geleff et al. in puromycin aminonucleoside nephrosis (PAN), a rat model of human minimal change nephropathy [10]. They found that the expression of PDPN was selectively reduced by $70 \%$ in PAN, which is characterized by the extensive flattening of podocyte foot processes and severe proteinuria. Subsequent studies showed that a single injection of anti-PDPN IgG into rats could induce selective proteinuria, which is paralleled by flattening of foot processes [11]. Zhang et al. further proved that osteocyte E11/gp38, which shares the same gene as PDPN, had a causal relationship with the dendrite formation of osteocyte-like cells [12]. Therefore, it is speculated that PDPN plays an important role in maintaining the normal morphology and function of podocytes.

Berberine (BBR), an isoquinoline alkaloid isolated from Coptis chinensis and Phellodendron chinense, has been used as an oral drug in traditional Chinese medicine to treat gastroenteritis and diarrhea for years [13]. Recent studies have indicated that BBR has multiple pharmacological activities, including glucose lowering, anti-inflammation, and anti-oxidation, suggesting that BBR has therapeutic efficacy against diabetes and its complications $[14,15]$. In the diabetic nephropathy mouse model, BBR ameliorated glomerulosclerosis through inhibition of the nuclear factor (NF)-KB signaling pathway [16]. In vitro experiments also showed that BBR could relieve podocyte injury and reduce high glucose $(\mathrm{HG})$-induced podocyte apoptosis by inactivating the

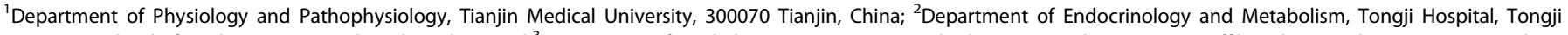

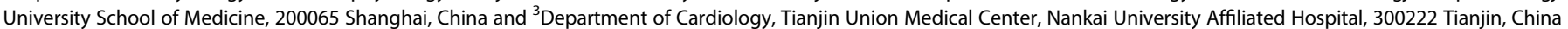
Correspondence: Ke-qin Zhang (keqzhang2018@163.com)

These authors contributed equally: Jing Yu, Guannan Zong

Received: 17 November 2018 Accepted: 22 May 2019

Published online: 3 July 2019 
NF-KB pathway [17]. However, whether PDPN is involved in the renoprotective effect of BBR remains unknown.

In this study, we used streptozotocin (STZ)-induced diabetic mice (a validated DKD model [18]) and HG-cultured mouse podocytes (MPC5 cells) to explore the role of PDPN in the development of DKD and the relationship between PDPN and the renoprotective effect of BBR.

\section{MATERIALS AND METHODS}

Chemicals and reagents

$\mathrm{STZ}$, berberine chloride (BBR), pyrrolidine dithiocarbamate (PDTC), dimethyl sulfoxide, trypsin, RPMI-1640 media and fetal bovine serum (FBS) were purchased from Sigma-Aldrich (St Louis, MO, USA). SYBR Green PCR Kit, BCA Protein Assay Kit, and mouse PDPN monoclonal antibody were purchased from Thermo Fisher Scientific (San Jose, CA, USA). Polyvinylidene difluoride (PVDF) membrane was purchased from Bio-Rad Laboratories (Hercules, CA, USA). Creatinine and Albumin Assay Kits were purchased from Nanjing Jiancheng Bioengineering Institute (Nanjing, China) and Beinglay Biotechnology Corporation (Wuhan, China), respectively.

\section{Animals}

Specific pathogen-free C57BL/6J male mice (8 weeks old, $20 \pm 2 \mathrm{~g}$ ) were purchased from Shanghai SLAC Laboratory Animal Center and housed in a conditioned atmosphere at a temperature of $24 \pm$ $2{ }^{\circ} \mathrm{C}$, with 12-h light/dark cycles and access to food and water ad libitum. The mice were acclimatized to housing conditions for 2 weeks prior to the experimental operation. All experimental procedures conformed to the China Animal Welfare Legislation and were reviewed and approved by the Tongji University Committee on Ethics in the Care and Use of Laboratory Animals.

\section{Cell culture}

Conditionally immortalized mouse podocytes (MPC5) were obtained from the Cell Resource Center of Peking Union Medical College (PUMC). The MPC5 cells were maintained and stimulated for proliferation and differentiation, as described previously [19]. Briefly, the MPC5 cells were first maintained in RPMI- 1640 medium supplemented with $10 \% \mathrm{FBS}$ and $10 \mathrm{U} / \mathrm{mL}$ recombinant mouse interferon- $\gamma$ (IFN- $\gamma$ ) for proliferation at $33^{\circ} \mathrm{C}$ in a humidified atmosphere of $5 \% \mathrm{CO}_{2}$. To induce cell differentiation, the cells were subcultured in RPMI-1640 medium (with 10\% FBS but without mouse IFN- $\gamma$ ) at $37^{\circ} \mathrm{C}$ for $10-14$ days, and the differentiated cells were used for subsequent experiments.

\section{Experimental design}

In this study, four sets of experiments were performed.

\section{Set1: Changes in PDPN expression levels and renal function} indicators during the progression of DKD

Sixty mice were randomly divided into the following groups: (1) normal control group $(n=30)$ and (2) STZ-induced diabetic group $(n=30)$. Both groups of mice were fasted for $12 \mathrm{~h}$ prior to injection, and mice in the STZ group then received intraperitoneal injections of $50 \mathrm{mg} / \mathrm{kg} \mathrm{STZ}$ diluted with sodium citrate buffer once daily for 5 consecutive days. At the same time point, the control group was injected with an equal volume of sodium citrate buffer. On the third day after STZ injection, the fasting blood glucose levels were examined in tail vein blood. When the blood glucose level reached $11.6 \mathrm{mM}$, the mice were confirmed to be diabetic [18]. During the experimental stage, 3 mice were randomly selected from each group and placed in metabolic cages to collect 24-h urine and blood samples for examination at 0, 2, 4, 8, 12, 16, and 20 weeks after the last STZ injection. Then the serum creatinine $(\mathrm{Scr})$ and urinary albumin levels of mice were detected using the Creatinine and Albumin Assay Kits, respectively. The kidney samples were also isolated for electron microscopic analysis and immunohistological examination after the mice were sacrificed.

Set2: Effects of HG stimulation on podocyte apoptosis and PDPN expression levels

In this experiment, we first explored the optimal conditions for inducing podocyte apoptosis with HG stimulation. We cultured MPC5 cells at different glucose concentrations $(5.6,12.5,30$, or 50 $\mathrm{mM}$ glucose) and different incubation times $(24,36,48 \mathrm{~h})$, and the proliferative activity of podocytes was determined using a CCK-8 assay. The results showed that the proliferative activity of MPC5 cells was affected by glucose in a dose- and time-dependent manner (Supplementary Fig. 1). Based on these data, we chose the concentration of $30 \mathrm{mM}$ and the time point of $36 \mathrm{~h}$ to conduct the following study.

To explore the effects of HG stimulation on podocyte apoptosis, we cultured MPC5 cells under different glucose concentrations and determined the apoptosis rate by flow cytometry. The MPC5 cells were randomly divided into three groups: (1) normal glucose (NG) group, (2) HG group, and (3) hypertonic control (HC) group. In the NG group, cells were incubated in RPMI 1640 medium supplemented with $5.6 \mathrm{mM}$ glucose. In the HG group, cells were incubated in RPMI-1640 medium supplemented with $30 \mathrm{mM}$ glucose. In the HC group, cells were incubated in RPMI-1640 medium supplemented with $30 \mathrm{mM}$ mannitol. The three groups were cultured at $37^{\circ} \mathrm{C}$ for 36 $\mathrm{h}$, and the percentage of apoptotic MPC5 cells in the different groups was determined.

To explore the effects of HG stimulation on the PDPN expression of podocytes, MPC5 cells were divided into three groups as above, i.e., the NG, HG, and HC groups. The MPC5 cells were cultured at $37^{\circ} \mathrm{C}$ for $36 \mathrm{~h}$, and PDPN mRNA levels were detected by real-time PCR.

Subsequently, interference and overexpression lentivirus vectors of PDPN were constructed and transfected into MPC5 cells. The apoptosis rates of podocytes with low or high expression of PDPN were determined under HG conditions so the effect of PDPN on podocytes could be clarified. To further explore the mechanism by which PDPN regulates cell apoptosis, the NF-KB signaling pathway of podocytes was also studied.

Set3: Protective effects of BBR on STZ-induced diabetic mice Sixty mice were randomly divided into two groups: the (1) normal control group $(n=30)$ and (2) STZ-induced diabetic group $(n=$ 30). The diabetic mouse model was induced with the same method as in Set1. After STZ injection, both groups were randomly divided into three groups each and received BBR treatment: the (1) control group $(n=10)$, (2) control group $+100 \mathrm{mg} / \mathrm{kg} \mathrm{BBR}(n=$ 10), (3) control group $+200 \mathrm{mg} / \mathrm{kg} \mathrm{BBR}(n=10)$, (4) diabetic group $(n=10)$, (5) diabetic group $+100 \mathrm{mg} / \mathrm{kg}$ BBR $(n=10)$, and (6) diabetic group $+200 \mathrm{mg} / \mathrm{kg}$ BBR $(n=10)$. Mice in all groups received $B B R$ treatment (intragastric administration) every day for 8 weeks. At the end of the experiment, 24-h urine, blood, and kidney samples were collected for examination.

Set4: The mechanism by which podocyte apoptosis is ameliorated by BBR under HG conditions

In this set of experiments, we first investigated the effects of different concentrations $(0,30,60,90$, or $120 \mu \mathrm{M})$ of $B B R$ on podocyte apoptosis under HG conditions. Based on the result that BBR relieved podocyte apoptosis in a dose-dependent manner, we chose the concentration of $90 \mu \mathrm{M}$ and the time point of $36 \mathrm{~h}$ to conduct the following study.

To further elucidate the mechanism by which podocyte apoptosis is ameliorated by BBR under HG conditions, we studied the activity of NF-KB pathway and PDPN protein levels in podocytes with or without BBR treatment using real-time PCR as well as Western blot. 
Electron microscopy analysis of mouse kidney tissue

Cortical kidney tissue samples were cut into $1 \mathrm{~mm}^{3}$ portions using a scalpel and then fixed in $2.5 \%$ glutaraldehyde for $2 \mathrm{~h}$. Then the sections were postfixed in $1 \%$ osmic acid for $2 \mathrm{~h}$ in a dark room. The specimens were dehydrated in a graded series of ethanol and embedded in Epon 812. After ultracutting, the ultrathin sections were stained with $1 \%$ uranyl acetate for $10 \mathrm{~min}$, followed by $2 \%$ Reynolds lead citrate buffer for $2 \mathrm{~min}$. Electron micrographs were analyzed on a Philips CM120 transmission electron microscope at $\times 4400$ magnification.

\section{Immunohistochemistry}

Kidney samples for immunohistochemical staining were fixed in $10 \%$ neutral-buffered formalin, embedded in paraffin, sliced into 4- $\mu \mathrm{m}$ sections, and placed onto microscope slides. Sections were deparaffinized by xylene, hydrated in decreasing concentrations of ethanol, and then quenched in $3 \% \mathrm{H}_{2} \mathrm{O}_{2} / 90 \%$ methanol solution for $10 \mathrm{~min}$ at room temperature to block endogenous peroxidase activity. To enhance antigen retrieval, sections were then subjected to high pressure in $0.01 \mathrm{mmol} / \mathrm{L}$ citrate buffer $(\mathrm{pH}$ 6.0) for $15 \mathrm{~min}$. After blocking with $5 \%$ bovine serum albumin for $30 \mathrm{~min}$ at room temperature to prevent nonspecific staining, sections were incubated with the primary antibody against PDPN $(1: 100)$ at $4{ }^{\circ} \mathrm{C}$ overnight in a humidified chamber. Following incubation with the horseradish peroxidase-conjugated secondary antibody for $30 \mathrm{~min}$ at room temperature, DAB chromogenic solution was added to the sections. When the color changed, the reaction was stopped with tap water. Finally, sections were counterstained with hematoxylin for $3 \mathrm{~min}$ and mounted for light microscopic evaluation. The relative expression level of PDPN was quantified using a microscope and Image-Pro Plus v6.0 software (Media Cybernetics, Silver Spring, MD, USA) according to the method described previously [20]. Briefly, an area of interest in each section was first selected at $\times 40$ magnification, and highresolution digital images were then captured at $\times 400$ magnification using microscopy and an image analysis system. The integrated optical density (IOD) was used to quantify the relative PDPN expression level.

\section{Quantitative real-time PCR}

Total RNA was extracted from MPC5 cells cultured under different concentrations of glucose using Trizol reagent (Invitrogen, Carlsbad, CA, USA) according to the manufacturer's protocol. Then first-strand cDNAs were synthesized from $1 \mu \mathrm{g}$ of total RNA using RevertAid First Strand CDNA Synthesis Kits (Fermentas, Amherst, NY, USA). Quantitative real-time PCR was performed using SYBR Green I as the detector dye. The relative expression level of PDPN mRNA was calculated by the $2^{-\triangle \triangle \text { ct }}$ method. The primer sequences used in this study were as follows: PDPN forward primer, 5'-GAGGGCTTAATGAATCTACTGGC-3' and reverse primer, 5'-GACAGTTCCTCTAAGGGAGGC-3'; GAPDH forward primer, 5'-CACCCACTCCTCCACCTTG-3' and reverse primer 5'-CCA CCACCCTGTTGCTGTAG-3'.

\section{Western blot assay}

Total protein was extracted using ice-cold RIPA lysis buffer (Solarbio, Beijing, China) supplemented with protease inhibitors (Roche, San Francisco, CA, USA), and the protein concentration was then measured by the BCA Protein Assay Kit (Thermo Fisher Scientific). Then $50 \mu \mathrm{g}$ of protein was electrophoresed on $10 \%$ sodium dodecyl sulfate-polyacrylamide gel electrophoresis for each sample and then transferred onto a PVDF membrane (BioRad, Hercules, CA, USA). The membrane was blocked with blocking buffer containing 5\% degreased milk powder for $1 \mathrm{~h}$ at room temperature, incubated with the primary antibody against PDPN (1:1000) at $4{ }^{\circ} \mathrm{C}$ overnight, detected with horseradish peroxidase-conjugated secondary antibody (1:10 000), and then visualized using enhanced chemiluminescent reagents $(E C L$,
Promega, USA). Densitometry analysis was performed with the ImageJ software. All experiments were repeated at least in triplicate.

Lentiviral vector construction and cell transfection

The lentiviral vectors were purchased from Sangon Biotech (Shanghai, China) to artificially interfere with or overexpress the PDPN gene. The coding sequence of mouse PDPN was acquired in $\mathrm{NCBI}$, and the targeting sequence of PDPN short hairpin RNA (shRNA) was 5'-GCGTGAATGAAGATGATAT-3'. Podocytes were transfected with lentiviral vectors according to the protocol provided by the manufacturer. The concentration of the lentiviral vector was $1 \times 10^{8}$ transducing units $/ \mathrm{mL}$, and polybrene (final concentration $5 \mu \mathrm{g} / \mathrm{mL}$ ) was added to enhance the lentiviral infection ratio. The podocytes were incubated for $96 \mathrm{~h}$ following transfection and then harvested using puromycin $(0.6 \mu \mathrm{g} / \mathrm{mL}$; Sigma-Aldrich) test. The living cells after puromycin selection were used for subsequent experiments.

\section{Data analysis}

The results are presented as the mean \pm SEM. Statistical analyses were carried out using SPSS 20.0 (IBM, Chicago, IL, USA). Differences between groups were analyzed by Student's $t$ test or one-way analysis of variance followed by Dunnett's test for multiple comparisons. Statistical significance was acceptable to a level of $P<0.05$.

\section{RESULTS}

Blood glucose, renal function, and podocyte ultrastructural changes in STZ-induced diabetic mice

To explore the early changes of the kidney in DKD, we employed the STZ-induced diabetic mouse model and examined the fasting blood glucose, renal function (including the Scr and albumin/ creatinine ratio $[A C R])$, and podocyte ultrastructural changes at 0 , $2,4,8,12,16$, and 20 weeks after the last STZ injection. As shown in Fig. 1a, mice in the control group had a normal blood glucose level of approximately $5 \mathrm{mM}$, while mice in the STZ group had blood glucose levels $>10 \mathrm{mM}$. In addition, we also observed that the Scr and ACR levels of the mice in STZ group were significantly higher than those in the control group 8 weeks after the last STZ injection $(P<0.05$, Fig. $1 \mathrm{~b}, \mathrm{c})$.

Correspondingly, the glomerular ultrastructure of mice in the STZ group also changed significantly from 4 weeks after STZ injection (Fig. 1d). In transmission electron microscopic images (magnification $\times 4400$ ) of both groups, we observed that the foot processes of podocytes in the STZ group were widened and fused at the 4th and 8th weeks (red arrows). At 12 weeks after STZ injection, the thickness of the glomerular basement membrane became nonuniform in the STZ group, and the normal structure of the glomerular filtration barrier almost completely disappeared. In contrast, the podocyte morphology in the control group remained normal during the whole observation period, and the nucleus and organelles could clearly be seen. These results indicated that mice in the STZ group suffered significant renal injury within 8 weeks after STZ injection.

Expression of PDPN in STZ-induced diabetic mice

We then sacrificed mice of each group at $0,2,4,8,12,16$, and 20 weeks after STZ injection for examination of kidney histology and the expression of PDPN. As shown in Fig. 2, PDPN was stained brown in the glomerulus, and the expression level of PDPN was represented by the IOD of the brown regions in the picture using the Image-Pro Plus 6.0 software (Media Cybernetics, USA). We observed a significant reduction of IOD in the STZ group from 8 weeks after STZ injection, which indicated that the expression level of PDPN was reduced with the development of DKD $(P<0.05$, Fig. 2b). However, 
a

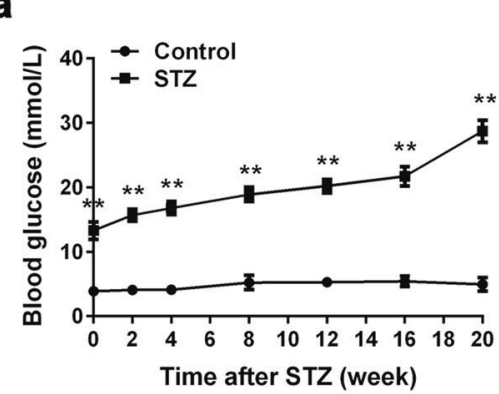

b

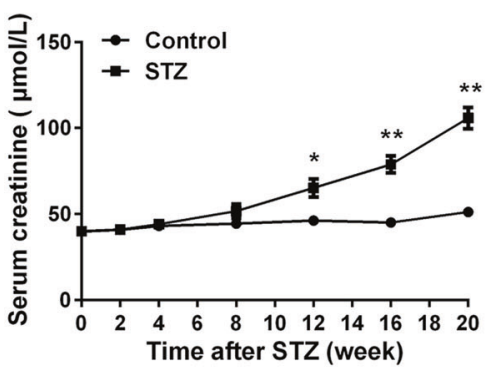

C

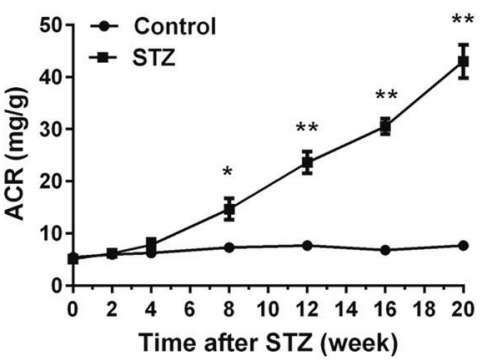

d
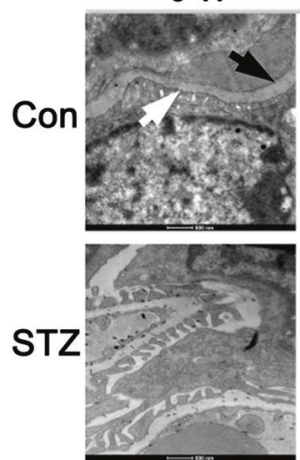

$2 \mathrm{~W}$

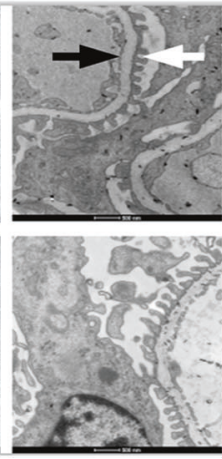

$4 \mathrm{~W}$

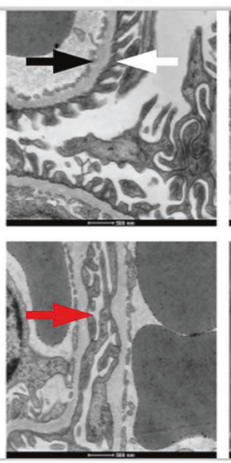

$8 \mathrm{~W}$

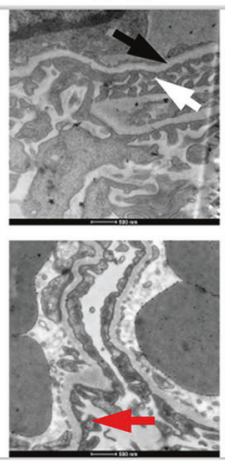

$12 \mathrm{~W}$

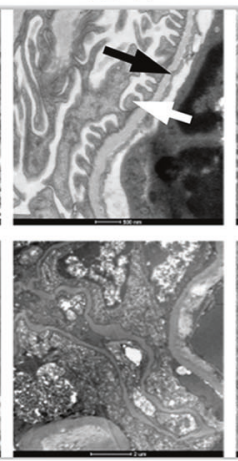

$16 \mathrm{~W}$

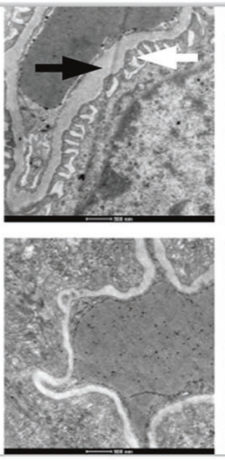

$20 \mathrm{~W}$

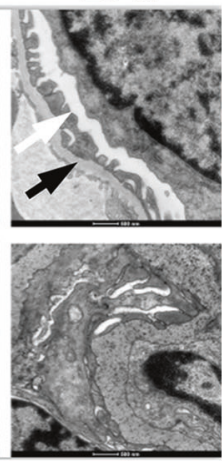

Fig. 1 Blood glucose (a), renal function (b, c), and podocyte ultrastructural changes (d) in streptozotocin (STZ)-induced diabetic mice. After mice were injected with STZ, fasting blood glucose level, renal function (including the serum creatinine and albumin/creatinine ratio level) were determined at different time intervals as indicated. To observe the ultrastructural changes of podocytes in early stages of diabetic kidney disease, electron microscopic examination was performed (magnification $\times 4400$ ). The arrows designate different structures of the glomerulus: white arrows, normal foot processes; black arrows, normal glomerular basement membrane; red arrows, fused foot processes. Con control group, STZ STZ-induced diabetic group. The data are expressed as the mean \pm SEM ( $n=3$ per time point). ${ }^{*} P<0.05,{ }^{* *} P<0.01$ compared to the normal control

the causal relationship between PDPN and podocyte injury was unclear.

Effects of HG stimulation on the apoptosis rate and PDPN expression of podocytes

To elucidate the relationship between podocyte injury and PDPN, we employed MPC5 cells under different glucose concentrations for $36 \mathrm{~h}$ and examined the apoptosis rate and PDPN expression level of MPC5 cells. The cells were divided into three groups: the (1) NG group (NG, $5.6 \mathrm{mM}$ glucose), (2) HG group ( $\mathrm{HG}, 30 \mathrm{mM}$ glucose), and (3) $\mathrm{HC}$ group ( $\mathrm{HC}, 30 \mathrm{mM}$ mannitol). The results demonstrated that the apoptosis rate of podocytes in the HG group was significantly higher than that in the NG group $(P<0.01$, Fig. $3 a, b)$. In addition, the PDPN mRNA level in the HG group was significantly downregulated compared with that in the NG group $(P<0.01$, Fig. 3c). Remarkably, we also demonstrated that high osmotic pressure had little effect on the apoptosis rate or PDPN expression level of podocytes (Fig. 3b, c).

Regulating the PDPN expression level can change the apoptosis rate of podocytes under HG conditions

To explore the causal relationship between PDPN and podocyte apoptosis under HG conditions, we synthesized PDPN shRNA and PDPN overexpression vectors and transfected them into MPC5 cells (Fig. 4a, b). First, we studied the effect of the low expression of PDPN on the apoptosis rate of podocytes by flow cytometry. The MPC5 cells were divided into four groups: the (1) control group (control, $5.6 \mathrm{mM}$ glucose), (2) HG group (HG, $30 \mathrm{mM}$ glucose), (3) control group+empty vector (Con+vector, $5.6 \mathrm{mM}$ glucose), and (4) control group+shRNA (Con+shRNA, $5.6 \mathrm{mM}$ glucose). The results showed that the apoptosis rate of podocytes in the HG group was significantly higher than that in the control group, which indicated that HG could induce podocyte apoptosis (Fig. 4c, e). In addition, we also observed that the apoptosis rate of podocytes with PDPN shRNA was significantly elevated even without HG stimulation (Fig. 4e). Based on these results, we hypothesized that low PDPN expression could induce podocyte apoptosis.

To further confirm this conjecture, we next artificially increased the PDPN expression level in podocytes under HG conditions to observe the changes of podocyte apoptosis. The MPC5 cells were divided into four groups: the (1) control group (control, $5.6 \mathrm{mM}$ glucose), (2) HG group (HG, 30 mM glucose), (3) HG+empty vector (HG+vector, $30 \mathrm{mM}$ glucose), and (4) HG+overexpression sequence ( $\mathrm{HG}+$ overexp, $30 \mathrm{mM}$ glucose). The PDPN protein levels and the apoptosis rate of podocytes were examined by Western blot and flow cytometry, respectively. The apoptosis rate of podocytes that overexpressed PDPN was significantly decreased compared with that of podocytes in the HG group $(P<0.01$, Fig. $4 d$, f). This result indicated that upregulating PDPN expression could reduce podocyte apoptosis induced by HG. Combined with the above results, PDPN expression levels were negatively correlated with the apoptosis rate of podocytes.

HG may inhibit PDPN expression by activating the NF-KB signaling pathway and thus induce the apoptosis of podocytes

We demonstrated that the expression level of PDPN is negatively correlated with the apoptosis rate of podocytes, but the mechanism of this phenomenon remained unclear. It has been reported that the inflammatory pathway mediated by NF-KB plays a pivotal role in the pathogenesis of renal disease in diabetes [21]. To investigate the relationship between HG stimulation and NF-KB activation, we employed the NF-kB inhibitor PDTC $(25 \mu \mathrm{M})$ and set up four groups: the (1) control group (control, $5.6 \mathrm{mM}$ glucose), (2) 
a
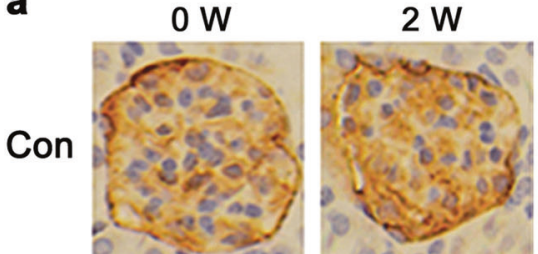

STZ

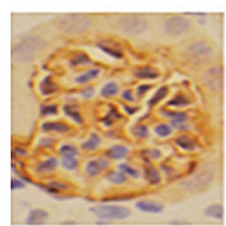

$4 \mathrm{~W}$
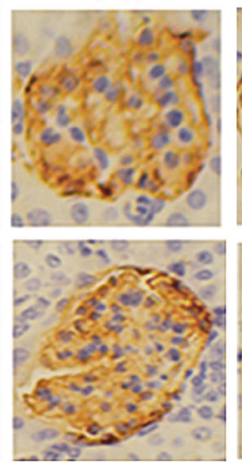

$8 \mathrm{~W}$
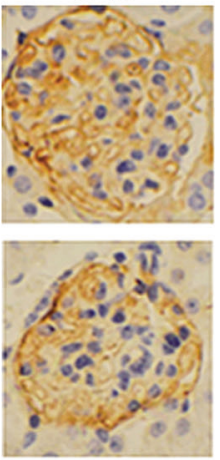

$12 \mathrm{~W}$
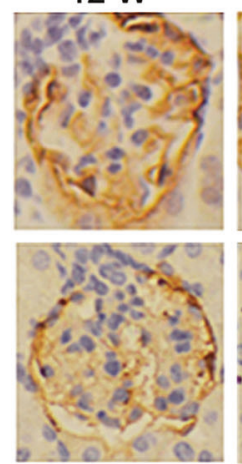

$16 \mathrm{~W}$
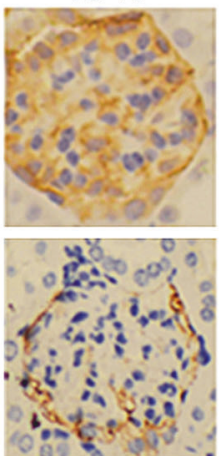

$20 \mathrm{~W}$
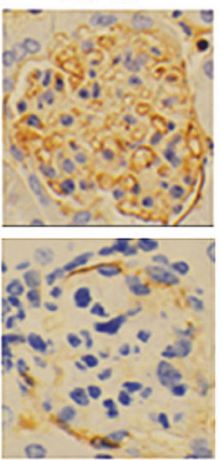

b

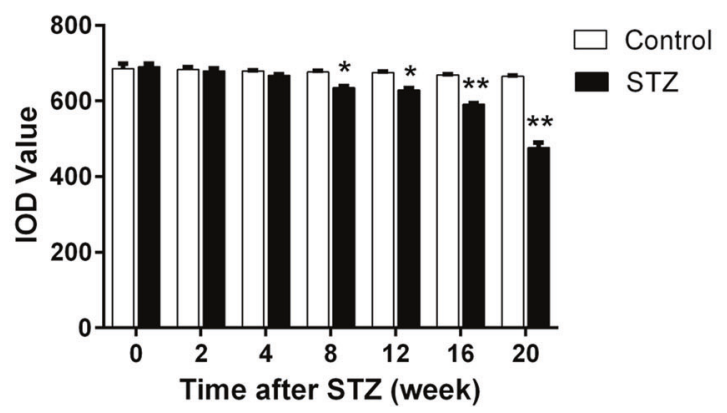

Fig. 2 Expression of podoplanin (PDPN) in streptozotocin (STZ)-induced diabetic mice. Kidney histology and expression of PDPN were documented with immunohistochemical staining (magnification $\times 400$ ). PDPN in the glomerulus stained brown, and the brown area of the STZ group was significantly smaller than that of the control group 8 weeks after STZ injection (a). To quantify the expression of PDPN, the integrated optical density (IOD) of the brown region in the picture was selected to represent the relative expression of PDPN (b). The data are expressed as the mean \pm SEM. ${ }^{*} P<0.05,{ }^{* *} P<0.01$ compared to the control group

a

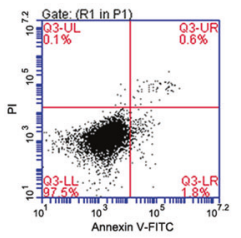

NG group

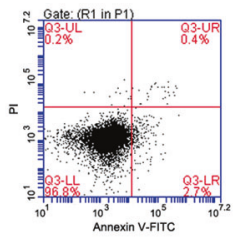

HC group

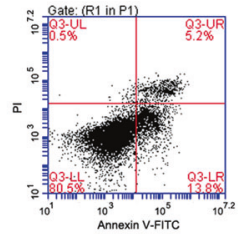

HG group
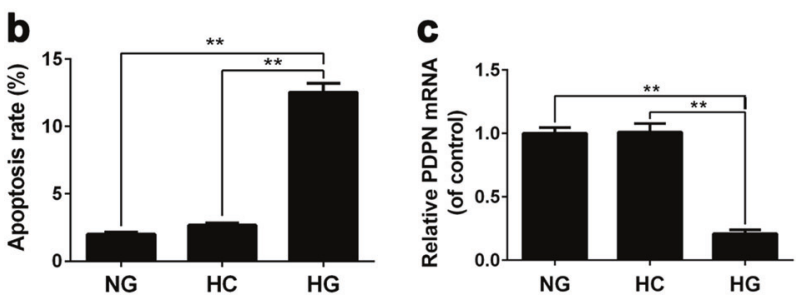

Fig. 3 The effects of high glucose stimulation on the apoptosis rate and podoplanin (PDPN) expression of podocytes. Cell apoptosis rates were analyzed using flow cytometry after $36 \mathrm{~h}$ of incubation in different glucose concentrations $(\mathbf{a}, \mathbf{b})$. The expression levels of PDPN mRNA of podocytes were examined by real-time PCR (c). ${ }^{* *} P<0.01$

control group+PDTC (Con+PDTC, $5.6 \mathrm{mM}$ glucose), (3) HG group (HG, $30 \mathrm{mM}$ glucose), and (4) HG group+PDTC (HG+PDTC, 30mM glucose). MPC5 cells were cultured in different concentrations of glucose for $36 \mathrm{~h}$, and the ratio of p-p65/p65 was then examined by Western blot. The results showed that the p-p65/p65 ratio of the HG group was significantly higher $(P<0.05)$ than that of the control group, while the $\mathrm{p}-\mathrm{p} 65 / \mathrm{p} 65$ ratio of the $\mathrm{HG}+\mathrm{PDTC}$ group was significantly decreased $(P<0.05)$ compared with that of the HG group (Fig. $5 \mathrm{a}, \mathrm{c}$ ). These results indicated that HG stimulation could activate the NF-KB signaling pathway.

To further explore the effects of the NF-kB signaling pathway on the PDPN expression and podocyte apoptosis, we set up four groups, which were the same as the above. The results showed that the expression level of PDPN in the HG group was significantly decreased $(P<0.01)$ compared with that in the control group, while the expression level of PDPN in the HG+PDTC group was significantly elevated $(P<0.01)$ compared with that in the HG group (Fig. 5b, d). This result indicated that suppressing the NF-KB signaling pathway could increase the PDPN expression level of podocytes under HG conditions. Similarly, we also observed that the podocyte apoptosis rate of the HG+PDTC group was significantly lower $(P<0.01)$ than that of the HG group (Fig. 5e, f), which indicated that suppressing NF-kB could reduce podocyte apoptosis under HG conditions. Based on these results, we speculated that HG may inhibit the expression of PDPN by activating the NF-KB signaling pathway and thus induce the podocyte apoptosis.

The amelioration of fasting blood glucose, renal function, and podocyte ultrastructure in diabetic mice treated with BBR By inducing diabetes with STZ, we observed that mice in the STZ group developed significant proteinuria within 8 weeks after the last STZ injection, which is considered to be the DKD state. Therefore, we used 60 mice treated with BBR from 8 weeks after the last STZ injection to observe the renoprotective effects of BBR. To ensure the therapeutic effect, the BBR treatment lasted for 8 weeks. As shown in Table 1, the fasting blood glucose of all STZ groups was significantly higher $(P<0.01)$ than that of the normal control group, but the increase of fasting blood glucose of mice in the STZ group was slowed down in a dose-dependent manner 

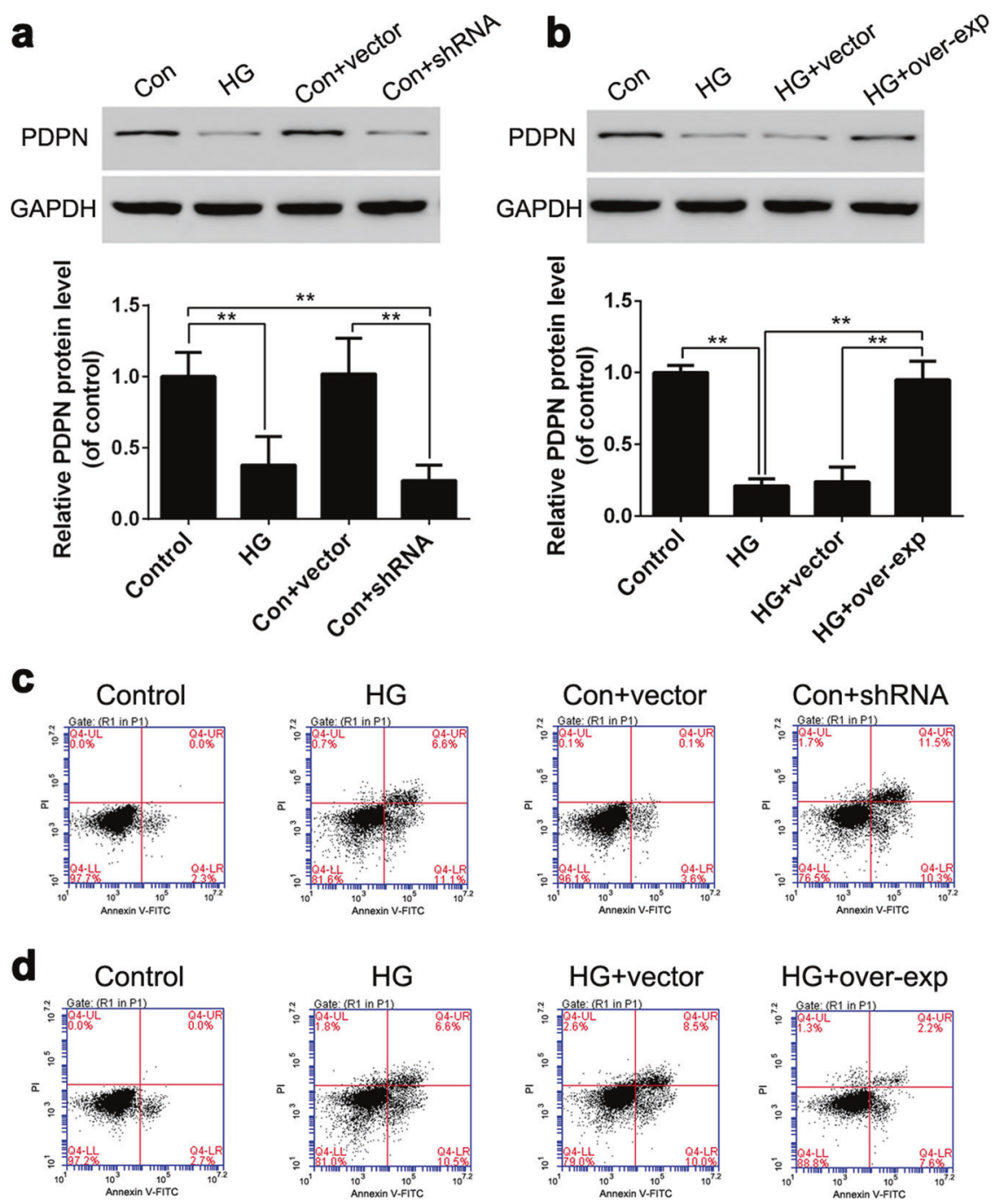

HG

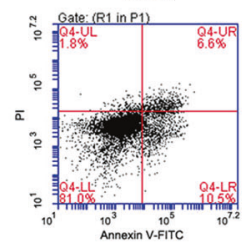

HG+vector
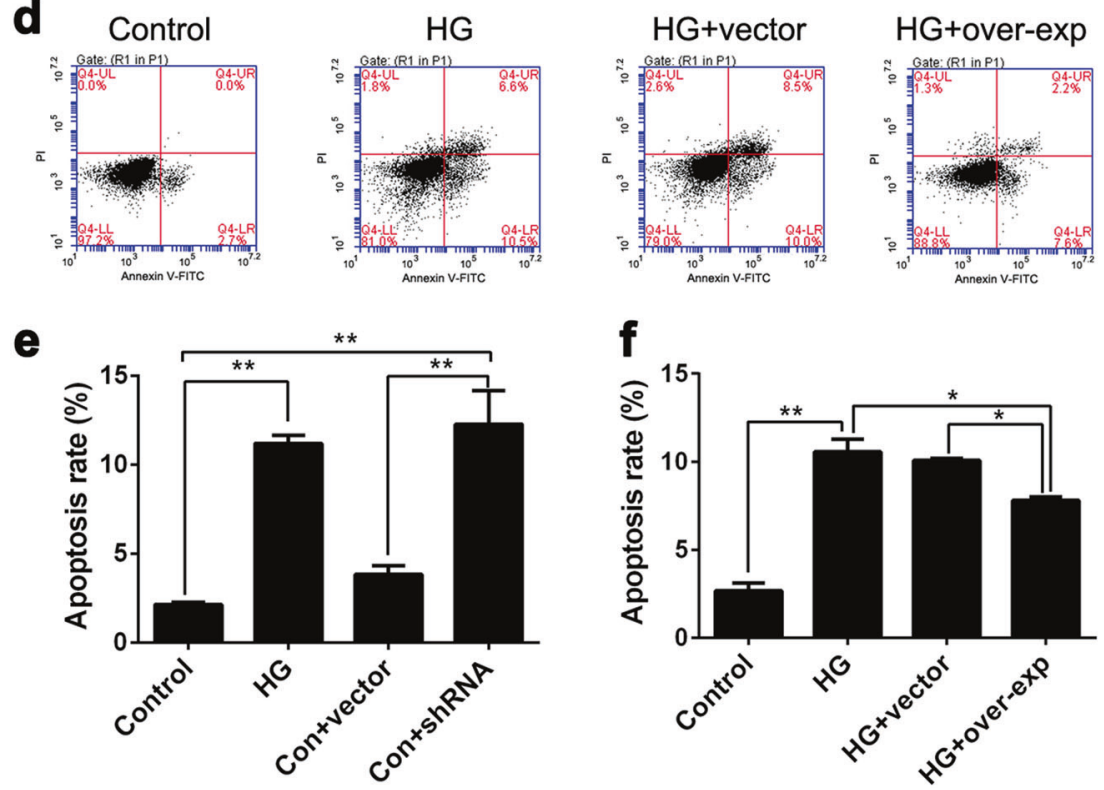

Fig. 4 Regulating podoplanin (PDPN) expression can change the apoptosis rate of podocytes cultured under high glucose conditions. To explore the relationship between PDPN expression and podocyte apoptosis under high glucose conditions, the PDPN shRNA sequences as well as overexpression sequence were transfected into MPC5 cells, and the PDPN protein levels of podocytes were successfully changed (a, b). The expression level of PDPN and the apoptosis rate of podocytes were examined by Western blot (a, b) and flow cytometry (c, d), respectively. The apoptosis rates of podocytes are shown in e, f. The data are expressed as the mean \pm SEM. ${ }^{*} P<0.05$, and ${ }^{* *} P<0.01$

after treatment with BBR. After 8 weeks of BBR treatment, the blood glucose levels of mice in the STZ $+100 \mathrm{mg} / \mathrm{kg}$ and STZ+200 $\mathrm{mg} / \mathrm{kg}$ groups were significantly decreased compared with mice in the STZ group. In addition, fasting blood glucose could not be decreased by intragastric administration of BBR in the control group, which indicated that BBR had little effect on the blood glucose of normal mice.
Similarly, the results also showed that BBR improved the renal function of diabetic mice. As shown in Fig. 6a, b, the Scr and ACR of mice in the STZ+100 mg/kg and STZ+200 mg/kg groups were significantly decreased compared with mice in the STZ group. For the ultrastructure of podocytes, the transmission electron microscopic analysis demonstrated that the podocyte foot processes of normal mice were long and thin, while those of diabetic mice 

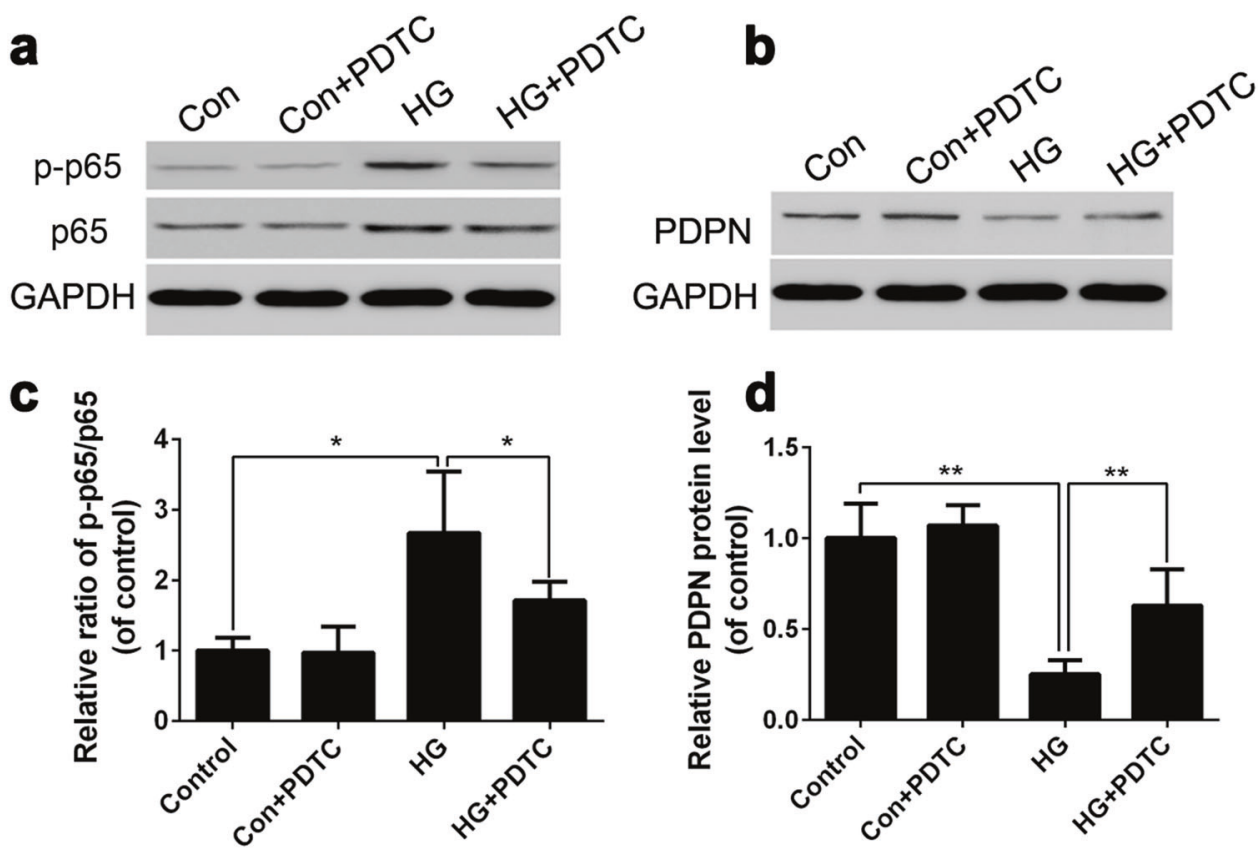

$\mathbf{e}$

Con

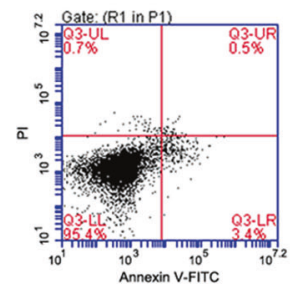

f

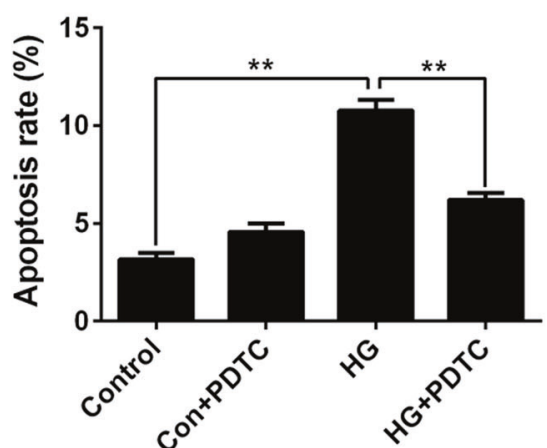

Con+PDTC
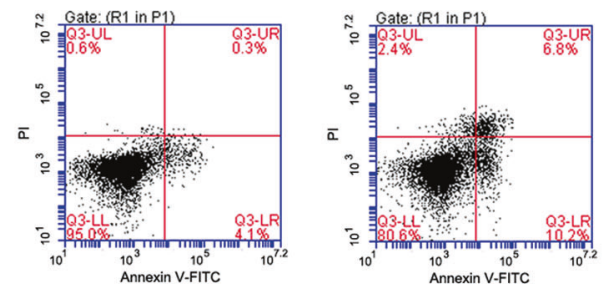

HG+PDTC

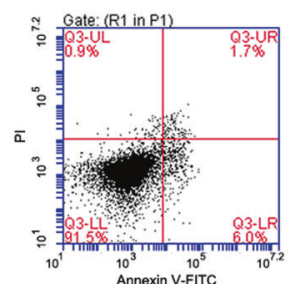

Fig. 5 Effects of the nuclear factor (NF)-кB signaling pathway on podoplanin (PDPN) expression and podocyte apoptosis under high glucose conditions. To explore the effects of the NF- $\mathrm{kB}$ signaling pathway on PDPN expression and cell apoptosis, the NF- $\mathrm{kB}$ inhibitor pyrrolidine dithiocarbamate was used. The protein levels of p65, p-p65, and PDPN were examined by Western blot (a, b), and the apoptosis rate was analyzed by flow cytometry (e). The relative ratio of p-p65/p65 and PDPN protein levels are shown in c, d, and the apoptosis rates of podocytes are shown in $\mathbf{f}$. The data are expressed as the mean \pm SEM. ${ }^{*} P<0.05,{ }^{*} P<0.01$

showed diffuse effacement and fusion. However, the foot process structure could be partially recovered after BBR treatment in the STZ groups (Fig. 6c).

BBR increases PDPN expression in STZ-induced diabetic mice After proving the renoprotective effects of BBR on STZ-induced diabetic mice, we further explored the effects of BBR on the expression of PDPN. Three mice were randomly selected from each group, and their kidney samples were isolated for immunohistochemistry. The results showed that the expression levels of PDPN in the STZ groups were significantly lower than those in the control groups. Furthermore, BBR treatment improved the PDPN expression level in a dose-dependent manner. As shown in Fig. 7, the dose of $200 \mathrm{mg} / \mathrm{kg}$ BBR could significantly increase the PDPN expression level compared with the untreated STZ group. In addition, BBR treatment had little effect on the PDPN expression of normal control mice.

The effects of BBR on the apoptosis rate and PDPN expression of podocytes under HG conditions

To further explore the mechanism of the renoprotective effects of BBR, we adopted MPC5 cells for research. The cells were cultured in $\mathrm{HG}$ medium containing different concentrations $(0,30,60,90$, or $120 \mu \mathrm{M})$ of BBR for $36 \mathrm{~h}$, the apoptosis rate 
Table 1. Fasting blood glucose levels in the control and STZ groups after BBR treatment

\begin{tabular}{llllllc}
\hline Time (week) & Control (mmol/L) & Con+100 (mmol/L) & Con+200 (mmol/L) & STZ (mmol/L) & STZ+100 (mmol/L) & STZ+200 (mmol/L) \\
\hline 0 & $4.21 \pm 0.43$ & $4.17 \pm 0.38$ & $4.33 \pm 0.43$ & $16.3 \pm 0.40^{\# \#}$ & $16.8 \pm 0.36^{\# \#}$ & $16.1 \pm 0.56^{\# \#}$ \\
2 & $5.13 \pm 0.45$ & $5.28 \pm 0.41$ & $4.78 \pm 0.40$ & $18.4 \pm 0.55^{\# \#}$ & $18.1 \pm 0.43^{\# \#}$ & $17.7 \pm 0.41^{\# \#}$ \\
4 & $5.33 \pm 0.35$ & $5.12 \pm 0.32$ & $4.12 \pm 0.28$ & $20.4 \pm 0.47^{\# \#}$ & $19.6 \pm 0.52^{\# \#}$ & $18.8 \pm 0.37^{* \# \#}$ \\
6 & $5.12 \pm 0.35$ & $5.32 \pm 0.48$ & $5.16 \pm 0.45$ & $22.6 \pm 0.90^{\# \#}$ & $20.9 \pm 0.37^{* \# \#}$ & $19.5 \pm 0.42^{* \# \#}$ \\
8 & $4.59 \pm 0.43$ & $5.31 \pm 0.45$ & $5.46 \pm 0.35$ & $25.1 \pm 0.67^{\# \#}$ & $21.2 \pm 0.43^{* * \# \#}$ & $20.2 \pm 0.58^{* * \# \#}$ \\
\hline
\end{tabular}

Data are presented as the mean \pm SEM ( $n=10$ per group)

Con control group, Con +100 control group with $100 \mathrm{mg} / \mathrm{kg} \mathrm{BBR,} \mathrm{Con+200} \mathrm{control} \mathrm{group} \mathrm{with} 200 \mathrm{mg} / \mathrm{kg}$ BBR, STZ STZ-induced diabetes group, STZ+100 STZ group with $100 \mathrm{mg} / \mathrm{kg} \mathrm{BBR}, S T Z+200 \mathrm{STZ}$ group with $200 \mathrm{mg} / \mathrm{kg} \mathrm{BBR}$

${ }^{*} P<0.05$ vs. STZ group; ${ }^{*} P<0.01$ vs. STZ group; ${ }^{\# \#} P<0.01$ vs. control group
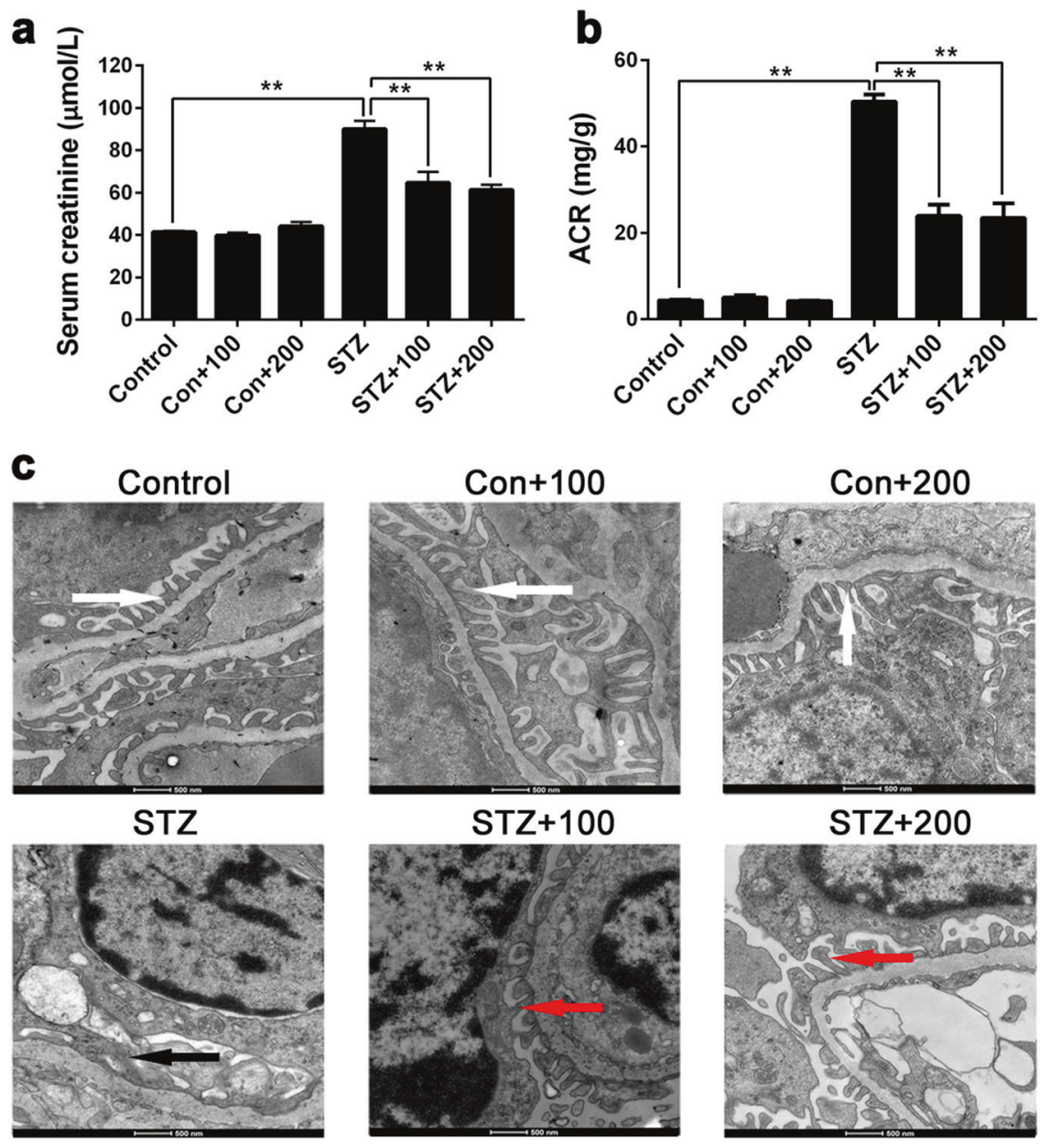

Fig. 6 The amelioration of renal function $(\mathbf{a}, \mathbf{b})$ and podocyte ultrastructure (c) in diabetic mice treated with berberine (BBR 100 and 200 mg/ $\mathrm{kg}$ ). Mice in both control and streptozotocin (STZ) groups received BBR treatment 8 weeks after the last STZ injection, and the BBR treatment lasted for 8 weeks. In the last week of the experiment, the renal function and podocyte ultrastructure were examined using detection kits and transmission electron microscopy (TEM; magnification $\times 4400)$. TEM analysis showed that the podocyte foot processes of the control groups were long and thin (white arrows), while those of the STZ groups showed diffuse effacement and fusion (black arrows). After BBR treatment, the structure of foot processes was partially recovered in the STZ groups (red arrows). The data are expressed as the mean \pm SEM ( $n=10$ per group). ${ }^{* *} P<0.01$

and PDPN expression levels were examined by flow cytometry and RT-PCR, respectively. The results showed that the apoptosis rate of MPC5 cells treated with BBR under HG conditions decreased significantly $(P<0.01)$ compared with untreated cells, and the decrease of apoptosis was dose-dependent (Fig. 8a, b). For the PDPN expression level, BBR could significantly $(P<0.01)$ increase the PDPN mRNA expression in MPC5 cells cultured in
HG medium when the concentration of BBR reached $60 \mu \mathrm{M}$ (Fig. 8c).

Effects of BBR on the activity of NF-KB signaling in podocytes cultured in HG medium

It has been reported that the inflammatory pathway mediated by NF-KB plays a pivotal role in the pathogenesis of renal disease in 

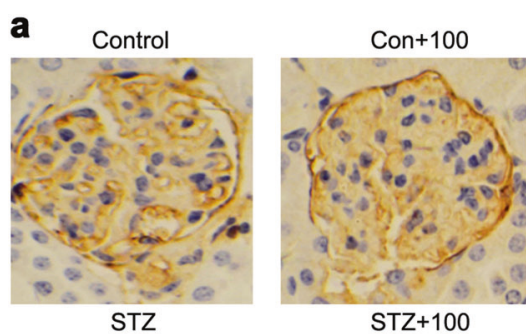

$\mathrm{STZ}+100$
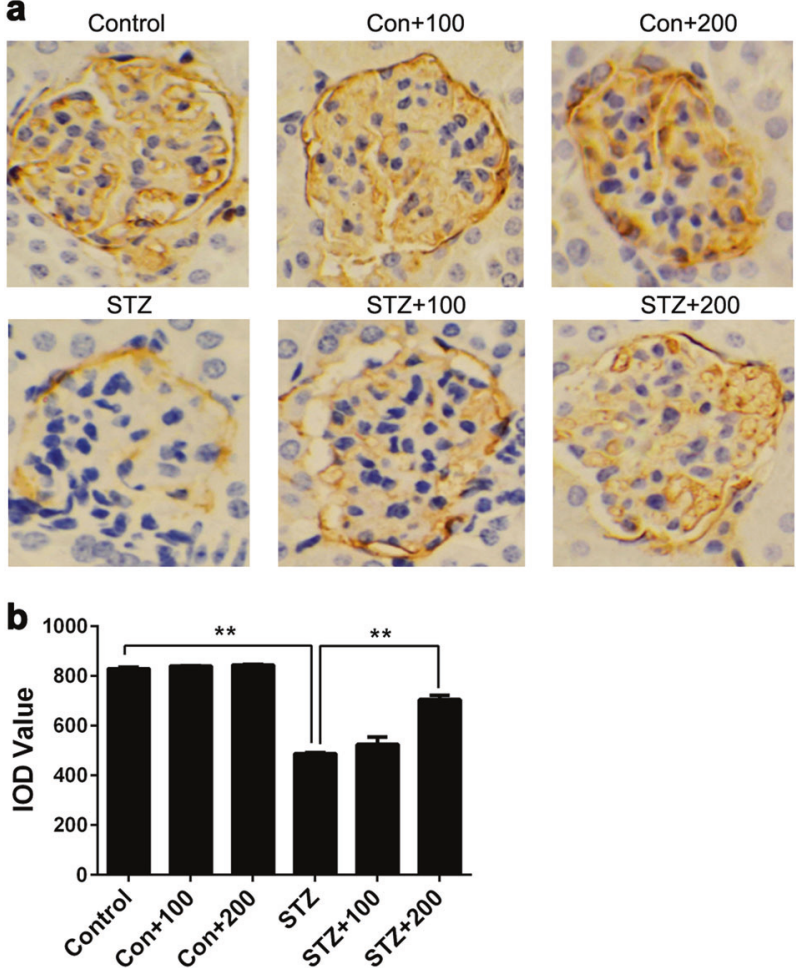

Fig. 7 Expression levels of podoplanin (PDPN) in streptozotocin (STZ)induced diabetic mice after BBR treatment. Kidney histology and PDPN expression levels were examined using immunohistochemical staining (magnification $\times 400$ ). PDPN in the glomerulus was stained brown, and the brown areas of the STZ groups were significantly smaller than those of the control groups (a). To quantify the expression level of PDPN, the integrated optical density (IOD) of the brown region in the picture was selected to represent the relative expression level of PDPN (b). Con +100 control group with $100 \mathrm{mg} / \mathrm{kg}$ BBR, Con +200 control group with $200 \mathrm{mg} / \mathrm{kg}$ BBR; STZ+100 STZ group with $100 \mathrm{mg} / \mathrm{kg}$ BBR, STZ+200, STZ group with $200 \mathrm{mg} / \mathrm{kg}$ BBR. The data are expressed as the mean \pm SEM. ${ }^{* * P} P<0.01$

diabetes [21]. To clarify whether BBR can inhibit the NF-KB signaling pathway, MPC5 cells were cultured in HG $(30 \mathrm{mM})$ medium supplemented with BBR solution $(90 \mu \mathrm{M})$ for $36 \mathrm{~h}$. The ratio of p-p65/p65 in podocytes was then examined by Western blot. The results showed that the p-p65/p65 ratio of the control group cells remained low with or without BBR treatment. In addition, the ratio of the $\mathrm{HG}+\mathrm{BBR}$ group was significantly lower $(P<0.01)$ than that of the HG group, which indicated that BBR could inhibit the activity of the NF-KB signaling pathway (Fig. 9a, C). In addition, the ratios of p-p65/p65 in the HG+BBR and $\mathrm{HG}+\mathrm{BBR}+$ shRNA groups were both lower than that of the $\mathrm{HG}$ group. This indicated that BBR could inhibit the activation of NF-KB induced by HG stimulation regardless of whether PDPN expression was blocked.

BBR promotes PDPN expression by inhibiting the NF-KB signaling pathway

To further clarify the mechanism by which BBR inhibits podocyte apoptosis under HG conditions, we set up six groups: the (1) control group (5.6 mM glucose), (2) control group+BBR (5.6 mM glucose $+90 \mu \mathrm{M}$ BBR), (3) HG group (30 mM glucose), (4) HG group + BBR (30 mM glucose $+90 \mu \mathrm{M}$ BBR), (5) HG group+BBR+PDPN shRNA ( $30 \mathrm{mM}$ glucose $+90 \mu \mathrm{M}$ BBR), and (6) HG group+vector ( $30 \mathrm{mM}$ glucose). The PDPN protein levels were examined by western blot. The results showed that the PDPN protein level of the HG group was significantly lower $(P<0.01)$ than that of the control group or HG+BBR group (Fig. 9b, d), which was consistent
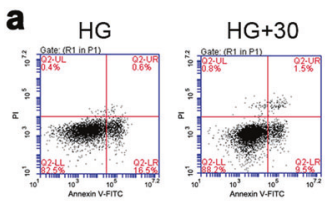

b

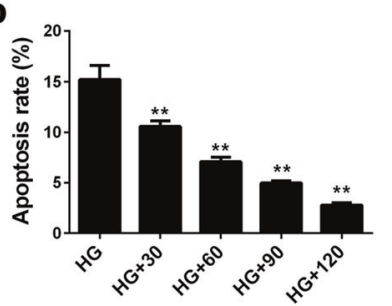

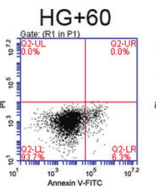
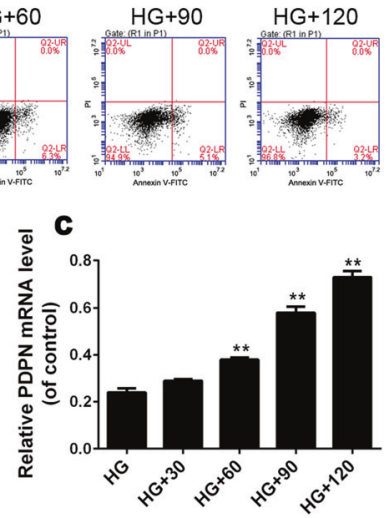

Fig. 8 The effects of berberine (BBR) on the apoptosis rate and podoplanin (PDPN) expression levels of podocytes under high glucose conditions. MPC5 cells were cultured in high glucose medium containing different concentrations of $\operatorname{BBR}(0,30,60,90$, or $120 \mu \mathrm{M})$ for $36 \mathrm{~h}$, and the apoptosis rate as well as PDPN expression levels was examined by flow cytometry $(\mathbf{a}, \mathbf{b})$ and reverse transcriptase-PCR (c), respectively. HG high glucose group $(30 \mathrm{mM}$ glucose), HG+30, HG group with $30 \mu \mathrm{M}$ BBR, HG+60, HG group with $60 \mu \mathrm{M}$ BBR, HG+90, HG group with $90 \mu \mathrm{M}$ BBR, $\mathrm{HG}+120, \mathrm{HG}$ group with $120 \mu \mathrm{M}$ BBR. The data are expressed as the mean \pm SEM. ${ }^{* *} P<$ 0.01 vs HG group

with previous experimental results (Fig. 5b, d). In addition, we also observed that the PDPN protein level of the HG+BBR+PDPN shRNA group was significantly decreased $(P<0.01)$ compared with that of the $\mathrm{HG}+\mathrm{BBR}$ group, which indicated that the BBR-induced PDPN upregulation disappeared when PDPN shRNA was transfected into podocytes. Therefore, the NF-KB pathway is located upstream of PDPN, and BBR can promote PDPN expression through inhibiting activation of the NF-KB pathway.

PDPN mediates the ability of BBR to inhibit podocyte apoptosis To further explore the mechanism by which BBR inhibits podocyte apoptosis under HG conditions, we set up the same groups as above and analyzed the cell apoptosis rate of each group. The results showed that the apoptosis rate of the HG+BBR group was significantly lower $(P<0.01)$ than that of the $\mathrm{HG}$ and $\mathrm{HG}+\mathrm{BBR}$ + shRNA groups (Fig. 9e, f). In combination with the previous experiments, we concluded that, when the PDPN expression of podocytes is inhibited, BBR can still inhibit the inflammatory pathway, but its antiapoptotic effect disappears. This result indicated that BBR inhibits activation of the NF-KB signaling pathway and thus increases the expression of PDPN to exert renoprotective effects.

\section{DISCUSSION}

DKD is one of the main causes of death for diabetic patients and is one of the most common complications of diabetes. Growing evidence has demonstrated that podocyte injury plays a pivotal role in the pathogenesis of DKD. PDPN, a type of membrane protein on podocytes, has at least two functions: (1) maintains cellular morphology and serves as a part of the glomerular mechanical barrier and (2) participates in the formation of the glomerular charge barrier with negative charge. Therefore, the normal expression of PDPN in podocytes is closely related to the glomerular filtration function.

In this study, we investigated the mechanism by which PDPN mediates the renoprotective effect of BBR in vivo and in vitro. First, we observed the changes of PDPN expression in STZ-induced diabetic mice and found that the PDPN levels decreased gradually along with the progression of diabetes. At the same time, the 

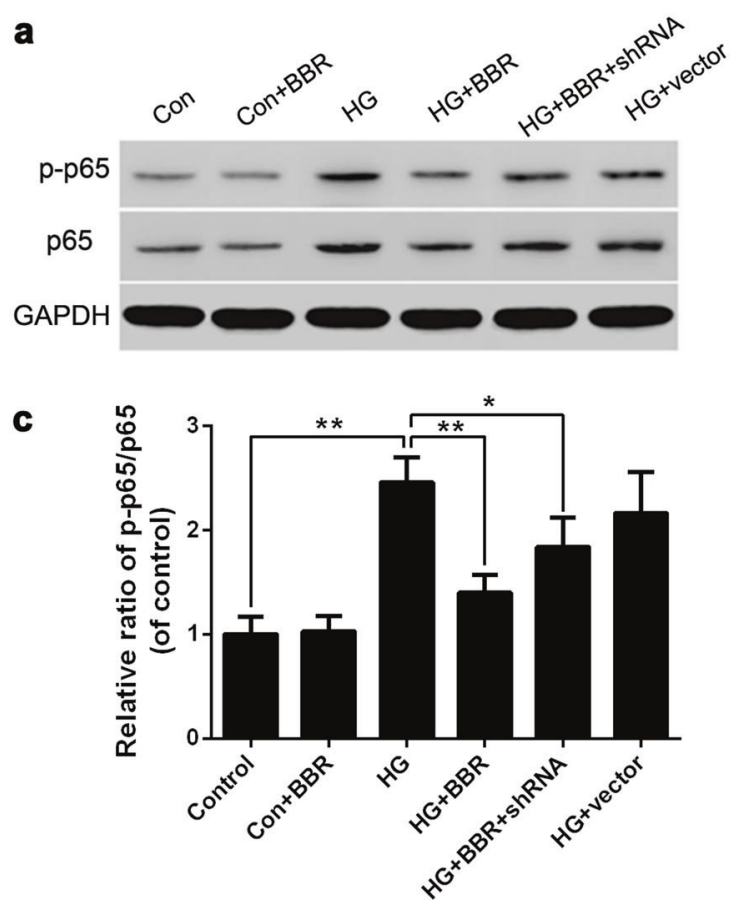

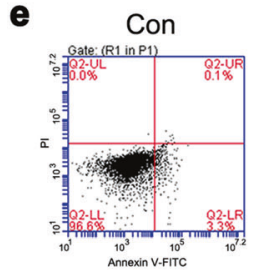

HG+BBR

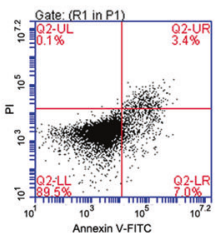

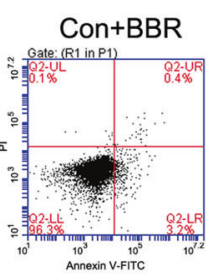

$H G+B B R+s h R N A$

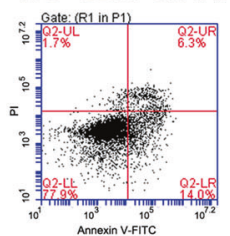

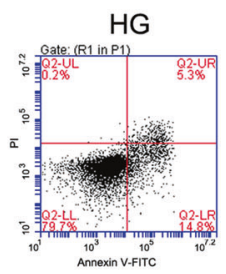

$H G+$ vector

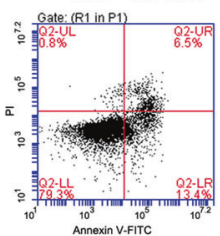

b

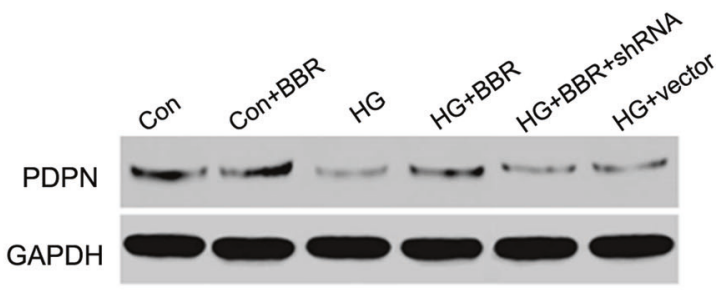

d

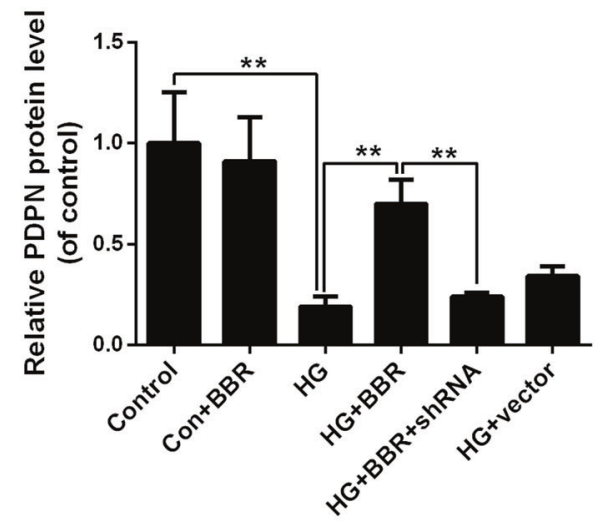

f

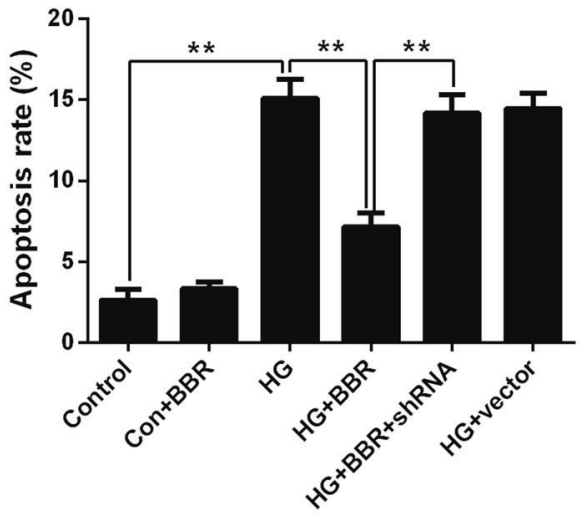

Fig. 9 The effects of berberine (BBR) on the apoptosis rate and podoplanin (PDPN) expression of podocytes cultured under high glucose conditions. MPC5 cells were cultured in normal glucose $(5.6 \mathrm{mM})$ medium, high glucose $(30 \mathrm{mM})$ medium, or high glucose $(30 \mathrm{mM})$ medium with $90 \mu \mathrm{M}$ BBR. Then p-p65/p65 protein level (a, c), PDPN protein level (b, d), and the apoptosis rates (e, $\mathbf{f})$ of MPC5 cells were analyzed by Western blot and flow cytometry, respectively. Control control group ( $5.6 \mathrm{mM}$ glucose), Con+BBR control group with $90 \mu \mathrm{M}$ BBR, HG high glucose group ( $30 \mathrm{mM}$ glucose), HG+BBR HG group with $90 \mu \mathrm{M}$ BBR, HG+BBR+shRNA HG group with $90 \mu \mathrm{M}$ BBR and PDPN shRNA, HG + vector HG group with the empty vector. The data are expressed as the mean \pm SEM. ${ }^{*} P<0.05,{ }^{* *} P<0.01$

podocyte ultrastructure as well as renal function indicators of diabetic mice also changed dramatically. However, the causal relationship between the decreased PDPN level and podocyte injury was not clear in vivo because of the long observation interval. To further explore this relationship, we cultured MPC5 cells in HG mediums. The results showed that there was increased apoptosis and decreased PDPN levels of podocytes under HG stimulation. In addition, we found that artificially regulating PDPN expression levels could influence the apoptosis rate of podocytes. More concretely, inhibiting PDPN levels of podocytes induced apoptosis even without HG stimulation, while overexpression of PDPN partially reversed the apoptosis induced by HG concentration. Therefore, we believe that there is a causal relationship between PDPN and podocyte apoptosis, and the apoptotic effect of HG on podocytes may be related to low expression of PDPN.

Although we described the relationship between PDPN and podocyte apoptosis, the specific mechanism by which
PDPN regulates podocyte apoptosis remained unclear. It has been reported that the inflammation mediated by NF-KB plays a key role in the pathogenesis of DKD [22]. In diabetic animals, the activation of NF-KB signaling is positively correlated with increased oxidative stress and inflammation, leading to renal dysfunction $[23,24]$. In our current study, the effect of NF-KB on PDPN expression and apoptosis of podocytes exposed to HG was investigated. The results showed upregulation of phosphorylated NF-KB p65, reduced PDPN levels, and increased podocyte apoptosis under HG conditions. However, after supplementing the medium with the NF-KB inhibitor PDTC, the activation of NF-KB was inhibited; meanwhile, podocyte apoptosis was reduced, and PDPN expression levels were increased. Taken together, these results indicate that HG inhibits the expression of PDPN by activating the NF-KB signaling pathway and thus induces podocyte apoptosis. 
Next, we explored the relationship between PDPN and the renoprotective effects of BBR. BBR is an alkaloid extracted from $C$. chinensis and $P$. chinense. It has been reported that BBR ameliorates renal function in diabetic mouse models [25]. In this study, BBR reduced blood glucose, ACR values, and Scr levels, and it also ameliorated the ultrastructure of podocytes in diabetic mice, indicating that BBR has therapeutic effects on DKD. Furthermore, in HG-cultured podocytes, BBR could inhibit activation of the NF-KB inflammatory pathway, increase PDPN levels, and reduce the apoptosis of podocytes exposed to HG. Interestingly, when the expression of PDPN was inhibited by shRNA, the antiapoptotic effect of BBR disappeared, although its inhibitory effect on NF-KB remained. These results indicate that the NF-KB pathway is located upstream of PDPN and that BBR promotes the PDPN expression via inhibition of the NF-KB signaling pathway to exert renoprotective effects. However, we also observed an unexpected result in the diabetic mouse model. Our results showed that the renoprotective effect of BBR at both doses was similar $(100,200 \mathrm{mg} / \mathrm{kg})$, but the expression levels of PDPN were different. Considering the hypoglycemic effect of BBR, the renoprotective effect of $B B R$ in mice may be due to its systemic effect by lowering blood glucose. However, the in vitro studies firmly proved the role of PDPN in BBR's renoprotective effect. In combination with the in vivo and in vitro studies, we propose that both the hypoglycemic effect and elevated PDPN expression level are involved in the mechanism of kidney protection by BBR.

In summary, our current study demonstrated that HG stimulation can activate the NF-KB signaling pathway, resulting in the low expression of PDPN, and can thus induce the apoptosis of podocytes. One of the mechanisms by which BBR ameliorates DKD is inhibiting activation of the NF-KB signaling pathway, thus restoring the expression of PDPN to reduce podocyte apoptosis induced by $\mathrm{HG}$ stimulation. These findings confirm the protective effect of BBR on podocytes and provide evidence for the therapeutic use of BBR for DKD.

\section{ACKNOWLEDGEMENTS}

This work was supported by the China Diabetes Young Scientific Talent Research Funding.

\section{AUTHOR CONTRIBUTIONS}

$\mathrm{KQZ}$ and JY contributed to the development of the project and experimental design, and JY, GNZ, and HW contributed to the performance of experiments and data analysis. All authors contributed to the paper writing and approved the final manuscript.

\section{ADDITIONAL INFORMATION}

The online version of this article (https://doi.org/10.1038/s41401-019-0263-3) contains supplementary material, which is available to authorized users.

Competing interests: The authors declare no competing interests.

\section{REFERENCES}

1. Foley RN, Collins AJ. The USRDS: what you need to know about what it can and can't tell us about ESRD. Clin J Am Soc Nephrol. 2013;8:845-51.
2. Reutens AT. Epidemiology of diabetic kidney disease. Med Clin North Am. 2013;97:1-18.

3. Ali IH, Brazil DP. Under the right conditions: protecting podocytes from diabetesinduced damage. Stem Cell Res Ther. 2013;4:119.

4. Gnudi L, Coward RJM, Long DA. Diabetic nephropathy: perspective on novel molecular mechanisms. Trends Endocrinol Metab. 2016;27:820-30.

5. Lal MA, Patrakka J. Understanding podocyte biology to develop novel kidney therapeutics. Front Endocrinol (Lausanne). 2018;9:409.

6. Dumont V, Tolvanen TA, Kuusela S, Wang H, Nyman TA, Lindfors S, et al. PACSIN2 accelerates nephrin trafficking and is up-regulated in diabetic kidney disease. FASEB J. 2017:31:3978-90

7. Liu Y, Su H, Ma C, Ji D, Zheng X, Wang P, et al. IQGAP1 mediates podocyte injury in diabetic kidney disease by regulating nephrin endocytosis. Cell Signal. 2019;59:13-23.

8. Huang N, Zhang X, Jiang $Y$, Mei H, Zhang L, Zhang Q, et al. Increased levels of serum pigment epithelium-derived factor aggravate proteinuria via induction of podocyte actin rearrangement. Int Urol Nephrol. 2019;51:359-67.

9. Fang J, Wei H, Sun Y, Zhang X, Liu W, Chang Q, et al. Regulation of podocalyxin expression in the kidney of streptozotocin-induced diabetic rats with Chinese herbs (Yishen capsule). BMC Complement Alter Med. 2013;13:76.

10. Breiteneder-Geleff $S$, Matsui $K$, Soleiman A, Meraner P, Poczewski H, Kalt R, et al. Podoplanin, novel 43-kd membrane protein of glomerular epithelial cells, is down-regulated in puromycin nephrosis. Am J Pathol. 1997;151:1141-52.

11. Matsui K, Breiteneder-Geleff S, Kerjaschki D. Epitope-specific antibodies to the 43$\mathrm{kD}$ glomerular membrane protein podoplanin cause proteinuria and rapid flattening of podocytes. J Am Soc Nephrol. 1998;9:2013-26.

12. Zhang K, Barragan-Adjemian C, Ye L, Kotha S, Dallas M, Lu Y, et al. E11/ gp38 selective expression in osteocytes: regulation by mechanical strain and role in dendrite elongation. Mol Cell Biol. 2006;26:4539-52.

13. Chen C, Yu Z, Li Y, Fichna J, Storr M. Effects of berberine in the gastrointestinal tract - a review of actions and therapeutic implications. Am J Chin Med. 2014;42:1053-70.

14. Xu L, Zhao W, Wang D, Ma X. Chinese medicine in the battle against obesity and metabolic diseases. Front Physiol. 2018;9:850.

15. Zhang X, Guan T, Yang B, Chi Z, Wan Q, Gu HF. Protective effect of berberine on high glucose and hypoxia-induced apoptosis via the modulation of HIF-1alpha in renal tubular epithelial cells. Am J Transl Res. 2019;11:669-82.

16. Liu W, Zhang X, Liu P, Shen X, Lan T, Li W, et al. Effects of berberine on matrix accumulation and NF-kappa B signal pathway in alloxan-induced diabetic mice with renal injury. Eur J Pharmacol. 2010;638:150-5.

17. Zhu L, Han J, Yuan R, Xue L, Pang W. Berberine ameliorates diabetic nephropathy by inhibiting TLR4/NF-kappaB pathway. Biol Res. 2018;51:9.

18. Tesch GH, Allen TJ. Rodent models of streptozotocin-induced diabetic nephropathy. Nephrology (Carlton). 2007;12:261-6.

19. Mundel P, Reiser J, Zuniga Mejia Borja A, Pavenstadt H, Davidson GR, Kriz W, et al. Rearrangements of the cytoskeleton and cell contacts induce process formation during differentiation of conditionally immortalized mouse podocyte cell lines. Exp Cell Res. 1997;236:248-58.

20. Xavier LL, Viola GG, Ferraz AC, Da Cunha C, Deonizio JM, Netto CA, et al. A simple and fast densitometric method for the analysis of tyrosine hydroxylase immunoreactivity in the substantia nigra pars compacta and in the ventral tegmental area. Brain Res Brain Res Protoc. 2005;16:58-64.

21. Liu R, Zhong $Y$, Li X, Chen $H$, Jim B, Zhou MM, et al. Role of transcription factor acetylation in diabetic kidney disease. Diabetes. 2014;63:2440-53.

22. Mora C, Navarro JF. The role of inflammation as a pathogenic factor in the development of renal disease in diabetes. Curr Diab Rep. 2005;5:399-401.

23. Yi B, Hu X, Zhang H, Huang J, Liu J, Hu J, et al. Nuclear NF-kappaB p65 in peripheral blood mononuclear cells correlates with urinary MCP-1, RANTES and the severity of type 2 diabetic nephropathy. PLoS One. 2014;9:e99633.

24. Kuhad A, Chopra K. Attenuation of diabetic nephropathy by tocotrienol: involvement of NFkB signaling pathway. Life Sci. 2009;84:296-301.

25. Zhang $X, \mathrm{He} \mathrm{H}$, Liang $D$, Jiang $Y$, Liang $W, C h i Z H$, et al. Protective effects of berberine on renal injury in streptozotocin (STZ)-induced diabetic mice. Int J Mol Sci. 2016;17:1327. 